\title{
Líquenes epífitos: una herramienta para estudiar la continuidad ecológica en Isla Mocha, Chile
}

\section{Epiphytic lichens: a tool for to study the ecological continuity Mocha Island, Chile}

\author{
Alejandro Gatica ${ }^{1}$, Iris Pereira ${ }^{2}$ \& Oscar Vallejos ${ }^{3}$ \\ ${ }^{1}$ Departamento de Biología, Universidad de La Serena, Casilla 599, La Serena, Chile. \\ ${ }^{2}$ Instituto de Biología Vegetal y Biotecnología, Universidad de Talca, Casilla 747, Talca, Chile. \\ ${ }^{3}$ Facultad de Ciencias Forestales, Universidad de Talca, Casilla 747, Talca, Chile. \\ alejandrogaticac@gmail.com; ipereira@utalca.cl; ovallejo@utalca.cl
}

\begin{abstract}
RESUMEN
El propósito de este trabajo fue estudiar la continuidad ecológica en la Reserva Nacional Isla Mocha, mediante el conocimiento de la riqueza y frecuencia de líquenes epífitos en 14 parcelas distribuidas al azar al interior de la reserva. La biomasa arbórea y volumen fustal fueron cuantificados para establecer correlaciones que pudieran explicar la distribución de líquenes epífitos. Se propone un nuevo índice de continuidad ecológica mediante cianolíquenes (ICEC) para conocer el grado de alteración de este ecosistema natural. La riqueza total de hongos liquenizados fue de 33 especies, de las cuales 3 constituyen nuevos registros para Chile: Fissurina triticea; Pyrenula neglecta y Sticta subcaperata. Se encontró una relación estadísticamente significativa entre cianolíquenes y el volumen fustal, lo que indicaría preferencia de este tipo de líquenes por zonas boscosas con un gran volumen fustal. Basado en el ICEC propuesto en este estudio, se definió a la Reserva Nacional Isla Mocha como un ecosistema alterado.
\end{abstract}

Palabras clave: Biomasa arbórea, índice de continuidad ecológica, líquenes epífitos, riqueza, volumen.

\begin{abstract}
The purpose of this work was to study the ecological continuity Mocha Island National Reserve, through the knowledge of the richness and frequency of epiphytic lichens in 14 plots distributed at random within the reserve. The tree biomass and upper-stem volume were quantified for to establish correlations that may explain the distribution of epiphytic lichens. It proposes a new index of ecological continuity with cyanolichens (ICEC) for to establish the degree of alteration of this natural ecosystem. The total richness of lichenized fungi was 33 species, 3 of which are new records for Chile: Fissurina triticea; Pyrenula neglecta and Sticta subcaperata. A statistically significant relationship was founded between cyanolichens and upper-stem volume, which could indicate preference of this type of lichens by forest ecosystem with a large upper-stem volume. In basis of the ICEC proposed in this work, Mocha Island National Reserve is defined as an altered ecosystem.
\end{abstract}

KEYWORDs: Index of ecological continuity, epiphytic lichens, richness, tree biomass, upper-stem volume.

\section{INTRODUCCIÓN}

El bosque es un conjunto funcional de recursos, donde la interacción entre la fauna, la flora, el suelo, el agua y el ambiente es clave, siendo éste una fuente de riqueza de especies, y de recursos económicos, estéticos y culturales (Ferretti 1997). Sin embargo, este tipo de recursos presenta una especial vulnerabilidad en superficies insulares, debido principalmente a la presión antropogénica (CONAF 2006).

Numerosos autores (Parmesan \& Yohe 2003, Root et al. 2003) han demostrado que los cambios producidos por efectos antrópicos, como la deforestación, desarrollo urbano y rural y tala del bosque han determinado fuertes cambios en la reproducción, la floración, la abundancia de plantas y animales y el anidamiento de diversas aves. En particular, alteraciones de la estructura del bosque y el incremento de las perturbaciones generan alteraciones en la composición y diversidad específica de las comunidades liquénicas (C.I. Rose 1976, F. Rose 1992).

La flora liquénica en la Reserva Nacional Isla Mocha ha sido escasamente estudiada, sólo se conocen especies de líquenes citadas por Galloway (1992), y recientemente un estudio preliminar de la flora liquénica (Quilhot et al. 2010), sin embargo, estos estudios sólo mencionan las especies 
presentes en la isla y no involucran cuestionamientos en relación con su representatividad.

La riqueza de líquenes epífitos ha sido usada para evaluar la continuidad ecológica de ambientes boscosos en particular en el hemisferio norte, esto ha permitido establecer un listado de especies liquénicas que permitan categorizar los bosques, basado en la presencia de un número dado de estos taxones, como es el caso del índice revisado de continuidad ecológica (RIEC) creado por Rose (1976). Dado que en Chile existe un bajo número de especies representadas en el listado de ese índice (7 de 30 especies), se optó por crear y proponer un nuevo índice de continuidad ecológica mediante cianolíquenes, dada la alta sensibilidad que estos líquenes poseen frente a diferentes factores de perturbaciones como contaminación del aire, perturbaciones propias del ecosistema como tala de bosque, presencia de pastoreo por ganado, desarrollo urbano en las cercanías de estos sistemas boscosos y cambio climático (Richardson 1992, Gries 1996, Ferretti 1997, Krivorotov 1998, Parmesan \& Yohe 2003, Richardson \& Cameron 2004, Cameron \& Richardson 2006).

De acuerdo a la información precedente, se plantearon en este trabajo los siguientes objetivos: a) conocer la riqueza y frecuencia de líquenes epífitos en los sectores norte y suroeste de la isla, b) relacionar la distribución de los líquenes con variables propias del bosque como el volumen fustal y la biomasa arbórea y c) establecer un nuevo índice en base a la frecuencia de cianolíquenes para evaluar la continuidad ecológica de los ecosistemas.

\section{MATERIALES Y MÉTODOS}

Área De Estudio

La Reserva Nacional Isla Mocha creada en 1988 forma parte del Sistema Nacional de Áreas Silvestres Protegidas del Estado (SNASPE), la cual se encuentra administrada por la Corporación Nacional Forestal (CONAF). Tiene una superficie de 2.182 ha y un rango altitudinal en el área muestreada entre 8 y $350 \mathrm{~m}$ s.n.m. Se encuentra ubicada en el Océano Pacífico, a $35 \mathrm{~km}$ al oeste de la desembocadura del río Tirúa, en las coordenadas $38^{\circ} 23^{\prime} \mathrm{S}, 73^{\circ} 52^{\prime} \mathrm{W}$. La vegetación superior asociada a la reserva es de similares características al bosque higrófilo valdiviano, pero con notoria ausencia del género Nothofagus. Las especies componentes del estrato arbóreo son principalmente: Aextoxicon punctatum Ruiz \& Pav.; Aristotelia chilensis (Molina) Stuntz; Fuchsia magellanica Lam.; Laurelia sempervirens (Ruiz \& Pav.) Tul.; Luma apiculata (DC.) Burret; Myrceugenia exsucca (DC.) O.Berg.; Peumus boldus Molina; Rhaphithamnus spinosus (Juss.) Moldenke, entre otras.

Isla Mocha se encuentra inserta en la región mediterránea perhúmeda (Di Castri 1968), con precipitaciones durante todo el año y medias anuales de $1.233 \mathrm{~mm}$, observándose un periodo de aridez producto del efecto barrera que proporciona el cordón montañoso. La temperatura media anual alcanza los $12,5^{\circ} \mathrm{C}$ (CONAF 2006). El bosque está relacionado desde un punto de vista biogeográfico con el bosque de neblina del norte chico y con el bosque templado valdiviano (Le Quesne et al. 1999, Mendoza \& Jerez 2001).

\section{CARACTERIZACIÓN DE LAS PARCELAS}

La obtención de los datos se realizó en octubre de 2008 en el sector norte y suroeste de la reserva, mediante un monitoreo en parcelas distribuidas al azar por los sectores más representativos de ésta, estableciéndose un total de 14 parcelas circulares de $200 \mathrm{~m}^{2}$ (7,98 $\mathrm{m}$ de radio) (Fig. 1), las cuales fueron clasificadas dentro de los cuatro siguientes sectores:

- Sector Laguna Hermosa (parcelas 1, 2, 3 y 4): presenta una longitud de 4,5 $\mathrm{km}$ con pendientes sobre el $40 \%$. La parcela 1 se caracteriza por poseer 5 individuos arbóreos jóvenes y todos corresponden a la especie Myrceugenia exsucca. Las parcelas 2 y 3 presentan 8 y 12 individuos arbóreos respectivamente, la mayor parte de ellos son árboles antiguos de la especie Aextoxicon punctatum. La parcela 4 tiene 10 individuos arbóreos antiguos entre los cuales hay 5 especies distintas.

-Sector Laguna Los Huairavos (parcelas 5 y 6): presenta una longitud de $400 \mathrm{~m}$ con pendientes fuertes sobre el $60 \%$. Estas parcelas están caracterizadas por una alta cobertura arbórea y baja luminosidad, en particular la parcela 5 presenta 10 individuos arbóreos con 3 especies distintas, mientras que la parcela 6 presenta 9 individuos con Aextoxicon punctatum como especie dominante.

-Sector Camino Nuevo (parcelas 7 y 8): presenta una longitud de $3.000 \mathrm{~m}$ con pendientes suaves. La parcela 7 presenta 8 individuos arbóreos de las especies Aextoxicon punctatum y Rhaphithamnus spinosus, en cambio la parcela 8 , que presenta un efecto borde por estar ubicada al límite de la Reserva Nacional, tiene 11 individuos y todos corresponden a Peumus boldus.

-Sector Sur Isla Mocha (parcelas 9, 10, 11, 12, 13 y 14): Se encuentran en una zona de ecotono, entre una zona de pastizal y arbustiva. Las parcelas presentan entre 6 y 15 individuos y como especies dominantes se encuentran Peumus boldus y Aristotelia chilensis. En particular la parcela 13 se encuentra en un bosque fragmentado con Peumus boldus como especie única.

Cada una de las parcelas fue georreferenciada mediante la proyección Universal Transversal de Mercator (UTM) y el Datum de referencia fue el sistema geodésico mundial de 1984 Huso 18 S (Tabla I). 


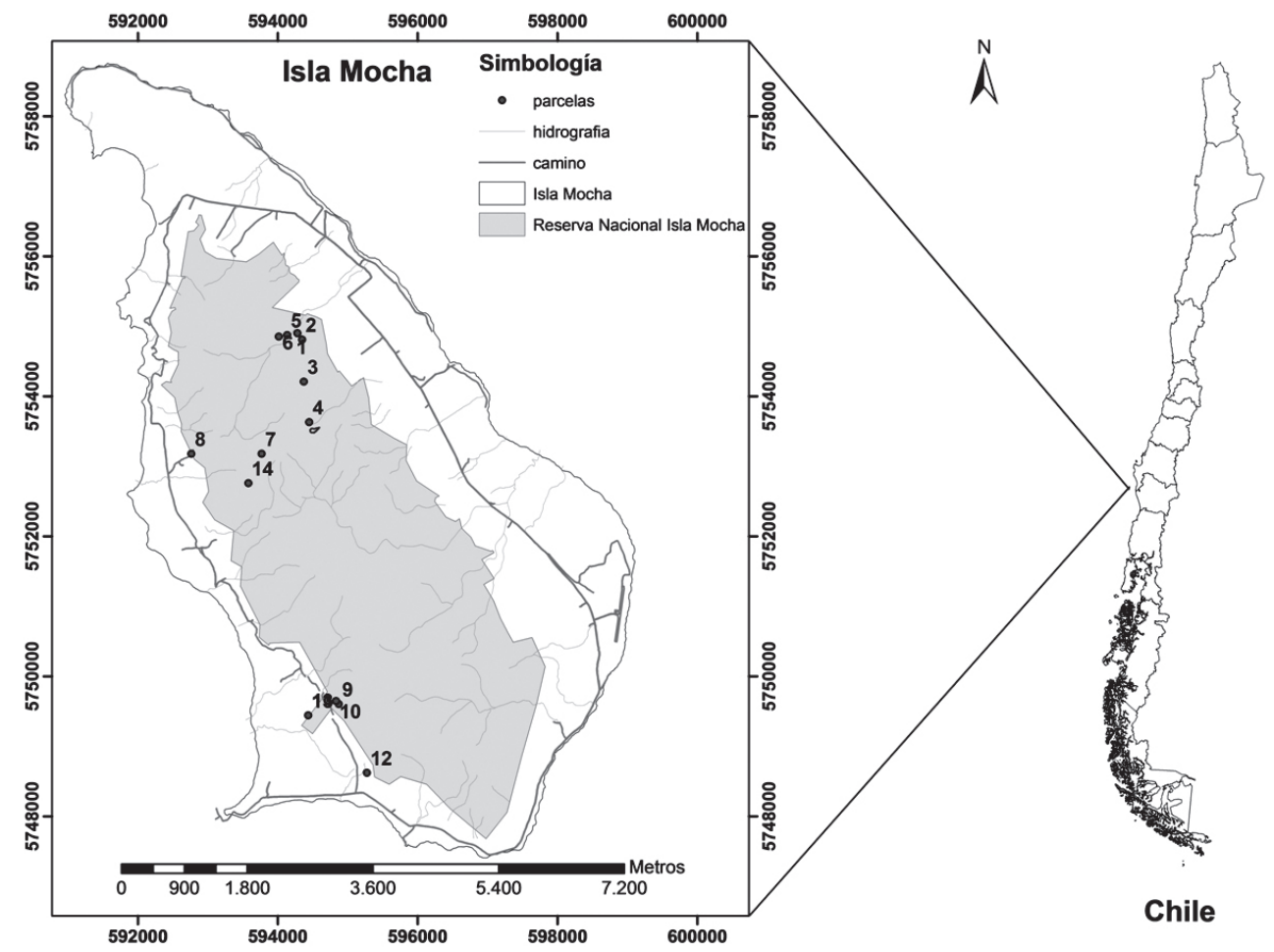

FiguRa 1. Distribución de las parcelas en la Reserva Nacional Isla Mocha.

FIgURE 1. Distribution of plots in the Mocha Island National Reserve.

TABLA I. Ubicación de parcelas y coordenadas UTM dentro de la Reserva Isla Mocha, Chile.

TABLE I. Location of plots and UTM coordinates into Mocha Island National Reserve, Chile.

\begin{tabular}{llll}
\hline PARCELA & Sector & \multicolumn{2}{c}{ CoORdENADAS UTM } \\
\hline 1 & Sendero Laguna Hermosa & 594282 & 5754900 \\
2 & Sendero Laguna Hermosa & 594348 & 5754808 \\
3 & Sendero Laguna Hermosa & 594372 & 5754209 \\
4 & Sendero Laguna Hermosa & 594449 & 5753631 \\
5 & Sendero Laguna Los Huairavos & 594136 & 5754872 \\
6 & Sendero Laguna Los Huairavos & 594014 & 5754852 \\
7 & Sendero camino nuevo & 593770 & 5754474 \\
8 & Sendero camino nuevo & 592764 & 5753178 \\
9 & Sector sur Isla & 594872 & 5749605 \\
10 & Sector sur Isla & 594834 & 5749648 \\
11 & Sector sur Isla & 594712 & 5749654 \\
12 & Sector sur Isla & 595276 & 5748622 \\
13 & Sector sur Isla & 594436 & 5749443 \\
14 & Sector sur Isla & 593580 & 5752759 \\
\hline & & & \\
\hline
\end{tabular}


MÉTOdo DE MUESTREO Y ANÁLISIS ESTADÍSTICOS

En cada una de las parcelas se determinó la riqueza y frecuencia de líquenes epífitos presentes en la corteza de los árboles, entre 0,5 y 2,0 m alt. La riqueza de líquenes se determinó cuantificando a los taxones disímiles presentes en la corteza de los árboles y la frecuencia fue la suma de todas las presencias de cada taxón por parcela.

Con la finalidad de conocer y establecer patrones de crecimiento local en la vegetación arbórea, se estimó la biomasa arbórea en toneladas (B: cantidad de materia orgánica originada a partir de la unidad física denominada árbol, incluye madera y hojas) y el volumen fustal en $\mathrm{m}^{3}$ ( $\mathrm{V}$ : magnitud física de un cuerpo en tres dimensiones: largo, ancho y alto).

Para estimar el volumen y biomasa de los árboles al interior de las parcelas se consideraron las siguientes variables: diámetro a la altura del pecho (D: 1,3 m alt.), el cual fue medido con una huincha diamétrica. La altura total del fuste $(\mathrm{H})$, medida con un clinómetro Suunto. El rendimiento volumétrico $\left(\mathrm{m}^{3}\right)$ y la biomasa (ton) de los árboles fueron estimados mediante modelos matemáticos específicos para cada especie arbórea (Tabla II). Los parámetros de dichos modelos no fueron ajustados en este trabajo, sino que fueron obtenidos basado en documentos técnicos descritos por Acuña et al. (2003), Gayoso et al. (2002) y Kannegiesser et al. (1998).

Los análisis estadísticos se realizaron con el software libre R (ver. 2.11.1) (R Development Core Team 2010) con ayuda del módulo de comandos RStudio (ver. 0.94.84), se realizaron análisis principalmente para observar correlaciones lineales y no paramétricas entre las variables biomasa arbórea, volumen fustal y riqueza liquénica.

\section{IDENTIFICACIÓN DE LAS ESPECIES}

La determinación de las especies de los hongos liquenizados se basó en un estudio de los caracteres macroscópicos, microscópicos e identificación de sustancias químicas. Los caracteres macroscópicos analizados fueron: medición del talo, apotecios, peritecios, mediante la ayuda de una lupa binocular Kyoma, modelo SDZ-PL. Para el análisis de los caracteres microscópicos se realizaron cortes a mano alzada de los apotecios y/o peritecios, practicados con una hoja de afeitar y montados en agua. Además se aplicaron reactivos como Lugol, ácido nítrico para reacciones específicas en el himenio o extremos de los ascos. Las mediciones de estructuras reproductivas internas se realizaron mediante una escala graduada dispuesta en el ocular del microscopio óptico Nikon modelo OPTIPHOT. La identificación de las sustancias liquénicas se llevó a cabo mediante test de coloración en la médula, cortex superior y talo usando pipetas capilares como medio de aplicación. Se usaron las siguientes soluciones: $\mathrm{K}$ : solución de hidróxido de potasio (KOH) 10\%, C: lejía comercial; KC: solución de hidróxido de potasio $(\mathrm{KOH})$, seguida de la aplicación de hipoclorito de sodio (lejía comercial) y finalmente P: solución alcohólica de parafenilendiamina (Clauzade \& Roux 1985).

Las especies fueron identificadas empleando literatura especializada, entre las cuales destacan: Wirth (1995a, b), Galloway, 1992, Kashiwadani (1990), Clauzade \& Roux (1985), Clauzade \& Ozenda (1970) y la nomenclatura sigue el Index Fungorum (Feuerer 2010).

De los líquenes identificados se guardan ejemplares de cada uno de los taxones en el Herbario del Instituto de Biología Vegetal y Biotecnología, aún sin acrónimo, Universidad de Talca, Talca, Chile.

\section{RESULTADOS}

RiQueZA LIQUÉNICA EN EL ÁREA ESTUDIADA En el área de estudio se registró una riqueza total de 33 especies de hongos liquenizados, de los cuales 3 son nuevos registros para el país: Fissurina triticea (Nyl.) Staiger (= Graphis triticea Nyl.); Pyrenula neglecta R.C.Harris y Sticta subcaperata (Nyl.) Nyl. Además se amplía la distribución de Pseudocyphellaria berteroana (Mont.) Redón para Chile insular.

Los géneros de líquenes observados con mayor frecuencia son los de hábito folioso, entre los cuales destacan Pseudocyphellaria, Sticta y Leptogium, donde el primero de éstos contribuye con un total de 7 especies. Los líquenes crustosos se encuentran representados por los géneros: Caloplaca, Fissurina, Graphis, Pertusaria y Pyrenula.

De las 33 especies registradas 6 corresponden a cianolíquenes y 27 a clorolíquenes, el detalle completo de las especies registradas se encuentra en la Tabla III.

RENDIMIENTO DEL BOSQUE ASOCIADO A LA RIQUEZA LIQUÉNICA Para establecer si existe alguna relación entre el rendimiento del bosque y la riqueza de líquenes se estimó la biomasa arbórea total y volumen fustal por parcela. Los principales resultados obtenidos a partir de la información colectada en terreno se resumen de la siguiente forma: Para la biomasa arbórea se obtuvo un promedio de 5,7 ton /parcela con una desviación estándar de 6,1; asimismo el volumen fustal presentó un promedio de $15,9 \mathrm{~m}^{3} /$ parcela con una desviación estándar de 18,3, en cambio la riqueza de líquenes promedio por parcela fue de 5 especies y una desviación de 3,8.

Dado que las variables biomasa y volumen fustal están fuertemente correlacionadas $\left(\mathrm{R}^{2}=0,99 ; \mathrm{P}=1,63 \mathrm{e}-12\right)$ (Fig. 2), se consideró para análisis posteriores solamente el volumen fustal, evitando así problemas de colinealidad en las estimaciones de los datos. Para mayores detalles de la relación existente entre la biomasa y volumen fustal se presenta la expresión [1].

Biomasa arbórea (ton) $=0,42845+0,33165 *($ Volumen arbóreo $\left.\left(\mathrm{m}^{3}\right)\right)$ 


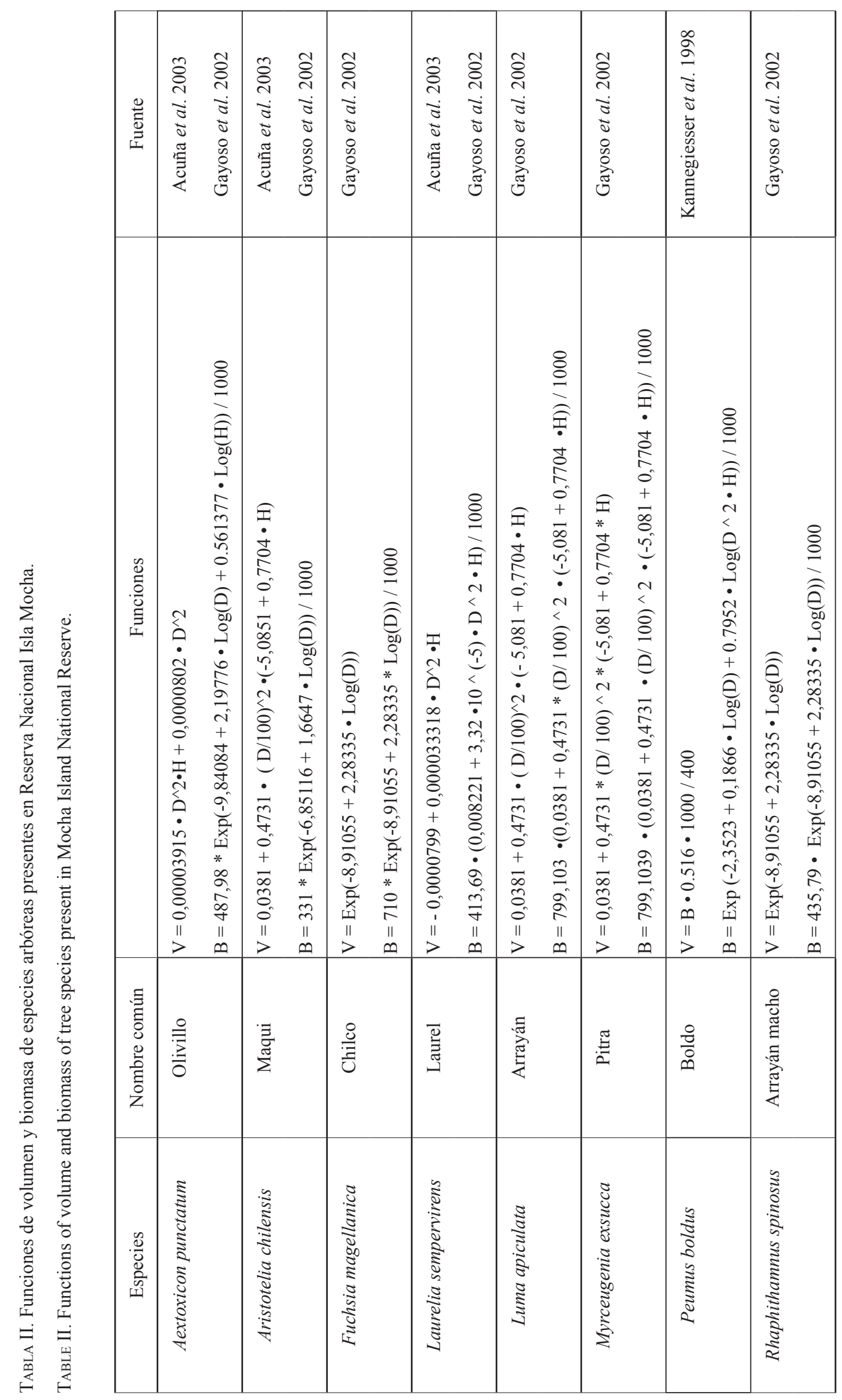


Líquenes epífitos de Isla Mocha: GaticA, A. ET AL.

TABLA III. Lista de especies, ficobionte y frecuencia de líquenes por parcela.

TABLE III. List of species, phycobiont and frequency of lichens per plot.

\begin{tabular}{|c|c|c|c|c|c|c|c|c|c|c|c|c|c|c|c|}
\hline \multirow[b]{2}{*}{ ESPECIES LIQUÉNICAS } & \multirow[b]{2}{*}{ FB } & \multicolumn{14}{|c|}{ Sitios de MUEstReo } \\
\hline & & 1 & 2 & 3 & 4 & 5 & 6 & 7 & 8 & 9 & 10 & 11 & 12 & 13 & 14 \\
\hline Bacidia laurocerasi (Delise ex Duby) Zahlbr. & $\mathrm{CL}$ & & & & & & & & & & & 2 & & & 1 \\
\hline Caloplaca citrina (Hoffm.) Th. Fr. & $\mathrm{CL}$ & & & & & & & & & & & & & 1 & \\
\hline *Fissurina triticea (Nyl.) Staiger & $\mathrm{CL}$ & & & & & & & & & 1 & & & & & \\
\hline Graphis scripta (L.) Ach. & $\mathrm{CL}$ & & & & & & & & 1 & & & & & & \\
\hline Hyperphyscia adglutinata (Flörke) H. Mayrhofer et Poelt & $\mathrm{CL}$ & & & & & & & & & & & & & 7 & \\
\hline Leptogium cochleatum (Dicks.) P.M.Jørg. \& P.James & CIA & & 5 & 7 & 4 & 2 & 3 & 5 & 6 & 6 & 4 & & & & 1 \\
\hline Parmotrema chinense (Osbeck) Hale \& Ahti & $\mathrm{CL}$ & & 1 & & & & & & 1 & & & & & 6 & \\
\hline Parmotrema crinitum (Ach.) M. Choisy & $\mathrm{CL}$ & & & & & & & & & & & & & 6 & \\
\hline Pertusaria leioplaca DC. & $\mathrm{CL}$ & & & & & & & & 2 & & & & & & \\
\hline Phaeophyscia orbicularis (Neck.) Moberg & $\mathrm{CL}$ & & & & & & & & & & & 3 & 1 & 6 & \\
\hline Physcia aipolia (Ehrh. ex Humb.) Fürnr. & $\mathrm{CL}$ & & & & & 1 & & & 2 & & & & & 7 & \\
\hline Physconia grisea (Lam.) Poelt & $\mathrm{CL}$ & & & & & & & & 7 & & & & & 4 & \\
\hline Pseudocyphellaria berberina (G. Forst.) D. J. Galloway \& P. James & $\mathrm{CL}$ & 1 & & 3 & & & 1 & & & & & & & & 1 \\
\hline **Pseudocyphellaria berteroana (Mont.) Redón & CIA & & & 1 & & & & 1 & & & & & & & \\
\hline Pseudocyphellaria coerulescens (Mont.) D.J. Galloway \& P. James & CIA & & & & & & & & & & & & & & 1 \\
\hline Pseudocyphellaria dissimilis (Nyl.) D. J. Galloway \& P. James & CIA & & & 1 & & & & & & & & & & & \\
\hline **Pseudocyphellaria freycinetii (Delise) Malme & $\mathrm{CL}$ & & & 2 & & & & & & & & & & & \\
\hline Pseudocyphellaria malmeana D. J. Galloway & $\mathrm{CL}$ & & & 3 & 6 & & & & & & & & & & 3 \\
\hline Pseudocyphellaria nudata (Zahlbr.) D. J. Galloway & CIA & & & & & & & & & & & & & & 2 \\
\hline Punctelia reddenda (Stirt.) Krog & $\mathrm{CL}$ & & & & & & & & 2 & & & & & & \\
\hline Punctelia subrudecta (Nyl.) Krog & $\mathrm{CL}$ & & & & & & & & & & & & & 10 & \\
\hline *Pyrenula neglecta R. C. Harris & $\mathrm{CL}$ & & & & & & & & & & 1 & & & & \\
\hline Pyrenula nitida (Weigel) Ach. & $\mathrm{CL}$ & & & 2 & & & & & 1 & & & & & & \\
\hline Ramalina striatula Ness \& Flot. & $\mathrm{CL}$ & & & & & & & & & & & & & 2 & \\
\hline Ramalina chilensis Bertero ex Nyl. & $\mathrm{CL}$ & & & & & & & & 2 & & & 2 & & & 1 \\
\hline Ramalina farinacea (L.) Ach. & $\mathrm{CL}$ & & & & & & & & 2 & & & & & & \\
\hline Sticta caliginosa D. J. Galloway & CIA & & 1 & 3 & 1 & 2 & 1 & 4 & & & 1 & & & & 1 \\
\hline *Sticta subcaperata (Nyl.) Nyl. & $\mathrm{CL}$ & & & 6 & 3 & & & & & & & & & & 2 \\
\hline Teloschistes flavicans (Sw.) Norman & $\mathrm{CL}$ & & & & & & & & 1 & & & & & & \\
\hline Teloschistes chrysophthalmus (L.) Beltr. & $\mathrm{CL}$ & & & & & & & & & & & & & 10 & \\
\hline Thelotrema lepadium (Ach.) Ach. & $\mathrm{CL}$ & & & & & & & & 1 & & 1 & & & & \\
\hline Usnea sp. & $\mathrm{CL}$ & 1 & & & & & & & 1 & & & & & & \\
\hline Xanthoria parietina (L.) Beltr. & $\mathrm{CL}$ & & & & & & & & & & & & & 13 & \\
\hline
\end{tabular}

* Nuevo registro para Chile (New records for Chile); ** Nuevo registro para Chile insular (New record for the insular flora of Chile); FB: Ficobionte (Phycobiont); CL: Clorofita (Chlorophyta); CIA: Cianofita (Cyanophyta). 


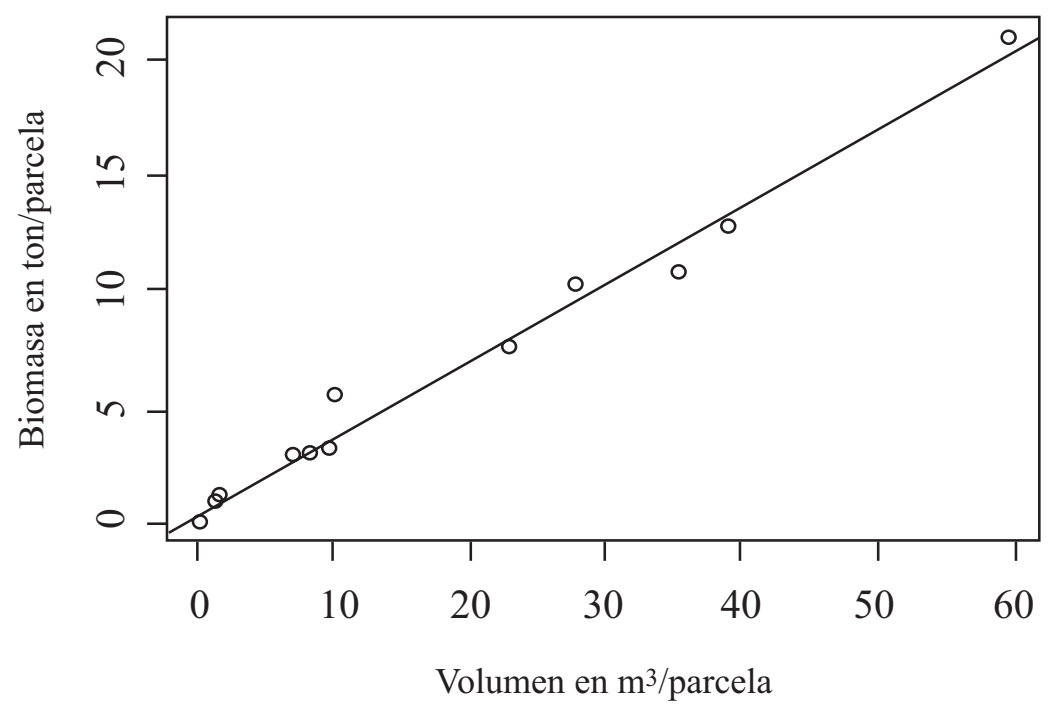

Figura 2. Correlación lineal entre el volumen fustal y la biomasa arbórea

FIGURE 2. Linear correlation between upper-stem volume and tree biomass.

Se estableció una correlación no paramétrica de Spearman entre la variable independiente "cianolíquenes" y la variable respuesta "volumen fustal". La relación entre estas variables está dada por un $\mathrm{r}=0,58$ y un $\mathrm{P}=0,0303$, estos resultados son estadísticamente significativos e indican que estas variables están moderadamente correlacionadas. Se realizó el mismo procedimiento para el volumen fustal y la frecuencia liquénica $(\mathrm{r}=-0,12 ; \mathrm{P}=0,6874)$ y para el volumen fustal y la riqueza liquénica $(\mathrm{r}=-0,06 ; \mathrm{P}=$ 0,8493). En ambos casos, el coeficiente de correlación no es estadísticamente significativo, lo cual indica que no existe una relación estadística clara entre estas variables.

ÍNDICE DE CONTINUIDAD ECOLÓGICA MEDIANTE CIANOLÍQUENES (ICEC)

Rose (1976) creó el índice revisado de continuidad ecológica (RIEC) para caracterizar viejos bosques estables o bosques no alterados, los cuales han mantenido una estabilidad ecológica por mucho tiempo. Este índice se basa en un conjunto de 30 especies de líquenes indicadoras, por lo tanto sólo es aplicable en un lugar donde se encuentren todas estas especies. En Chile se encuentran 7 de las 30 especies descritas por Rose (1976), las cuales se detallan a continuación: Chaenotheca brunneola (Ach.) Müll.Arg.; Pannaria rubiginosa var. vulcanica Zahlbr.; Parmotrema crinitum (Ach.) Choisy; Peltigera collina (Ach.) Schrad.; Porina leptalea (Durieu \& Mont.) A.L. Sm.; Sticta limbata (Sm.) Ach.; Thelotrema lepadinum (Ach.) Ach. (Feuerer 2010). De estas 7 especies citadas para Chile sólo 2 se encuentran citadas para la Isla Mocha.

Debido al escaso número de especies liquénicas indicadoras de bosques estables en la isla, se propone una modificación al índice presentado por Rose (1976), el cual se señala a continuación

El índice de continuidad ecológica mediante cianolíquenes (ICEC), el cual se representa en la expresión 2.

ICEC $=$ (Frecuencia de Cianolíquenes / Frecuencia liquénica)*100

Este índice plantea el uso de cianolíquenes como indicadores de alteraciones ambientales, este tipo de indicadores justifica su inclusión por estudios realizados por Hallingback (1989), Richardson (1992) y Krivorotov (1998), en los cuales se han registrado declinaciones de cianolíquenes producto de alteraciones antrópicas. Por su parte, Gries (1996), Wirth (1988) describen a los cianolíquenes como agentes biológicos sensibles a los contaminantes del aire. Mientras que un estudio realizado por Richarson \& Cameron (2004) ha demostrado que un bajo número de cianolíquenes en ecosistemas oceánicos o en bosques antiguos estarían indicando algún grado de alteración ambiental debido a la destrucción del hábitat provocada en gran parte por las prácticas forestales inadecuadas.

A partir de la información obtenida en terreno, las parcelas fueron clasificadas basado en el valor del ICEC, cuyos valores se encuentran disponibles en la Tabla V, en la cual se puede observar que 5 parcelas se encuentran en zonas muy alteradas, 3 en zonas alteradas, 1 levemente alterada y 5 sectores no alterados. Además se calculó un ICEC para la Reserva Nacional, dicho valor fue de 32,5, el cual clasifica a la reserva como un bosque alterado. 
Líquenes epífitos de Isla Mocha: GaticA, A. ET AL.

TABLA IV. Categorización para aplicación de índice de continuidad ecológica.

TABLE IV. Categorization for the application of ecological continuity index.

\begin{tabular}{cl}
\hline $\begin{array}{c}\text { Índice de Continuidad Ecológica } \\
\text { Mediante cianolíquenes (ICEC) }\end{array}$ & \multicolumn{1}{c}{ Categorías } \\
\hline $0-24,9$ & Bosque muy alterado \\
$25-49,9$ & Bosque alterado \\
$50-74,9$ & Bosque levemente alterado \\
$75-100$ & Bosque no alterado \\
\hline
\end{tabular}

TABLA V. Principales resultados obtenidos por ICEC en las parcelas muestreadas al interior de la Reserva Nacional Isla Mocha.

TABLE V. Main results obtained by ICEC in the sampled plots within the Mocha Island National Reserve.

\begin{tabular}{ccl}
\hline PARCELA & ICEC & \multicolumn{1}{c}{ CATEGORÍA } \\
\hline 1 & 0 & Bosque muy alterado \\
2 & 85,7 & Bosque no alterado \\
3 & 42,9 & Bosque alterado \\
4 & 35,7 & Bosque alterado \\
5 & 80 & Bosque no alterado \\
6 & 88,9 & Bosque no alterado \\
7 & 100 & Bosque no alterado \\
8 & 20,7 & Bosque muy alterado \\
9 & 85,7 & Bosque no alterado \\
10 & 71,4 & Bosque levemente alterado \\
11 & 0 & Bosque muy alterado \\
12 & 0 & Bosque muy alterado \\
13 & 0 & Bosque muy alterado \\
14 & 38,5 & Bosque alterado \\
\hline
\end{tabular}

\section{DISCUSIÓN}

En este estudio se suman tres nuevos registros para Isla Mocha y el país: Fissurina triticea (Nyl.) Staiger (= Graphis triticea Nyl.), Pyrenula neglecta R. C. Harris y Sticta subcaperata (Nyl.) Nyl., aparte de los ya previamente presentados por Quilhot et al. (2010).

Las variables volumen fustal y cianolíquenes presentaron una correlación no paramétrica moderada $(\mathrm{r}=0,58)$, pero con significancia estadística $(\mathrm{p}=0,0303)$, sin embargo, hay que considerar el número de parcelas reducido de este estudio. Por lo tanto, un mayor número de parcelas podría encontrar una relación más fuerte en un nuevo estudio.

El bosque de la Reserva Nacional Isla Mocha es de características muy húmedas, similares al bosque higrófilo valdiviano, sin embargo la presencia de cianolíquenes en la isla es escasa, a pesar de la influencia oceánica y la antigüedad del bosque (Cameron \& Richardson 2006). Esto podría deberse a la fuerte competencia por la humedad y espacio físico entre cianolíquenes y briófitos (Sillett \& McCune 1998) y/o la degradación paulatina del bosque, mediante la tala indiscriminada para consumo por parte de la población local, quienes hacia 1980 requerían de unos 1000 árboles adultos para ser usados como cierres de potreros, vivienda y combustible (Pefaur \& Yáñez 1980).

El ICEC puede ser de utilidad para evaluar ecosistemas naturales a gran escala (macrohábitats), sin embargo, si se aplica este índice a escalas pequeñas el valor puede ser afectado por variables ambientales tales como: efecto de borde y la sombra en el fuste de los árboles (Brodo 1973, 
Hale 1955). Según Werth (2001) los factores más importantes que influirían en la composición de macrolíquenes epífitos serían en orden de importancia gradientes macroclimáticos, variación espacial y variaciones en el sustrato.

A nivel de microhábitats, la variabilidad de los valores del índice podría deberse según (Brodo 1973, Hale 1955), a alteraciones ambientales, $\mathrm{pH}$ de la corteza de los árboles, contenido mineral, luz y temperatura, siendo claves para explicar la sustitución de especies liquénicas. En conclusión, cada tipo de árbol presentaría características propias que podrían facilitar o no la presencia o ausencia de líquenes epífitos.

En este estudio en particular, la parcela 1 no presenta cianolíquenes, por lo tanto su ICEC fue cero, clasificándose como un bosque muy alterado, lo cual podría deberse a la presencia sólo de 5 individuos arbóreos y todos ellos correspondientes a la misma especie (Myrceugenia exsucca), sin embargo esta parcela se encuentra muy cerca de las parcelas 2, 5 y 6 , las cuales presentan un bosque no alterado según el índice, lo que podría responder a una heterogeneidad del $\mathrm{pH}$ de los árboles y a un mayor número de especies arbóreas dentro de estas parcelas. La ausencia o baja frecuencia de cianolíquenes, en las parcelas $8,11,12$ y 13, se puede explicar por el efecto borde, generando una mayor luminosidad y temperatura en estas parcelas, lo cual influiría en una baja frecuencia de cianolíquenes, donde la presencia de sus pigmentos accesorios (ficobilínicos) presentes en sus fotobiontes les otorgaría la posibilidad de sobrevivir en sitios de baja luminosidad y temperatura.

Las parcelas georreferenciadas, el registro de la riqueza y frecuencia de líquenes epífitos son datos que facilitarán establecer comparaciones que permitan advertir cambios en la dinámica de estas comunidades liquénicas en monitoreos a largo plazo. Esto permitirá un seguimiento de la entidad ambiental a través del tiempo, observando el estado de conservación y la fenología los líquenes, en respuesta a un estímulo bien definido como lo indica Stevens (1994). De acuerdo a lo anterior, se recomienda un monitoreo periódico de la riqueza y frecuencia liquénica en las parcelas ya establecidas, con el fin de evaluar la continuidad ecológica en el ecosistema.

A falta de una lista de especies liquénicas indicadoras de la estabilidad de ecosistemas boscosos, como la propuesta por Rose (1976), se espera que el índice de continuidad ecológica, mediante cianolíquenes (ICEC) propuesto, pueda ser aplicado en diversos ecosistemas similares, definiendo así el grado de alteración ambiental en ambientes con influencia oceánica o en bosques antiguos y así tomar las medidas para su conservación (Ederra 1996).

Se recomienda la aplicación del ICEC sólo en ecosistemas con influencia oceánica o en bosques antiguos, debido a que estos ambientes son ideales para el desarrollo de cianolíquenes. Además se plantea la comparación del resultado de este índice con la Tabla IV para establecer el grado de alteración del ecosistema en estudio.

\section{AGRADECIMIENTOS}

Se agradece el apoyo económico brindado por el Programa de Investigación de Productos Bioactivos, IQR y al IBVB, Universidad de Talca y a CONAF por el apoyo logístico brindado durante la estadía en la Reserva Nacional.

\section{BIBLIOGRAFÍA}

Acuña, E., P. Emanuelli \& F. Drake. 2003. Compendio de funciones dendrométricas del bosque nativo. Universidad de Concepción. Santiago, Chile. 197 pp.

Brodo, I. 1973. Substrato Ecology. In: V. Ahmadjian \& M.E. Hale (eds.), The Lichens: 401-440. Academic Press. New York. London.

Cameron, R. \& D. Richardson. 2006. Occurrence and abundance of epiphytic cyanolichens in protected areas of Nova Scotia, Canada. Opuscula Philolichenum 3: 5-14.

Clauzade, G. \& P. Ozenda. 1970. Les Lichens: Étude biologique et flore illustrée. Editorial Masson et cie., Paris 802 pp.

Clauzade, G. \& C. Roux. 1985. Likenoj de okcidenta europo. Ilustrita determinlibro. Bulletin de la Société Botanique du Centre-Ouest. Niort, Francia. 893 pp.

CONAF (Corporación Nacional Forestal, Chile). 2006. Reserva Nacional Isla Mocha: Biodiversidad; Conservación; Cultura, Cañete, Chile. 131 pp.

Di Castri, F. 1968. Equisse écologique du Chili. Biologie de l'Amerique australe. In: C.L. Debouteville \& E. Rapaport (eds.), Etude sur la faune du Sol. Vol. IV: Biologie de Lamerique Australe: 7-52. Editions du Centre National de la Recherche Scientifique, Paris, France.

Ederra, A. 1996. Botánica ambiental aplicada: Las plantas y el equilibrio ecologico de nuestra tierra. Universidad de Navarra, Pamplona, España. 205 pp.

Ferretti, M. 1997. Forest health assessment and monitoring - issues for consideration. Environmental Monitoring and Assessment 48: 45-72.

Feuerer, T. 2010. Checklist of lichens and lichenicolous fungi of Chile. Version 1 June 2010. Url: http://www.biologie.unihamburg.de/checklists/lichens/south-america/chile_l.htm Visto: Junio 25, 2011.

Galloway, D. 1992. Studies in Pseudocyphellaria (lichens) III: The South American species. Bibliotheca Lichenologica 46: $1-275$.

Gayoso, J., J. Guerra \& D. Alarcón. 2002. Contenido de carbono y funciones de biomasa en especies nativas y exóticas. Universidad Austral de Chile, Proyecto medición de la capacidad de captura de carbono en bosques de Chile y promoción en el mercado mundial. Informe Final, Documento $\mathrm{N}^{\mathrm{o}}$ 1, Valdivia, Chile. $53 \mathrm{pp}$.

GrIEs, C. 1996. Lichens as indicators of air pollution. In: T.H. Nash III (ed.), Lichen Biology. Cambridge University Press, Cambridge 63(8): 364-367.

Hale, M.E., JR. 1955. Phytosociology of corticolous cryptogams 
in the upland forests of southern Wisconsin. Ecology 36: 45-6.

HallingBäck, T. 1989. Occurrence and ecology of the lichen Lobaria scrobiculata in southern Sweden. Lichenologist 21: 331-341.

Kannegiesser, U., R. Rosende \& M. Toral. 1998. Biomasa y boldina en boldo (Peumus boldus Mol.) VII región. Ciencia e Investigación Forestal 2(4): 15-25.

Kashiwadani, H. 1990. Some Chilean species of the genus Ramalina (Lichens). Bulletin of the National Science Museum 16: 1-12.

Krivorotov, S. 1998. Change of coverage and number of Lobaria pulmonaria (L.) Hoffm. due to anthropogenic pollution in the mountain forests of the north-western Caucasus. pp. 112-113. In: S.Y. Kondratyuk, B.J. Coppins (eds.), Lobarion Lichens as Indicators of the Primeval Forests of the Eastern Carpathians. 192 pp.

Le Quesne, C., C. Villagrán \& R. Villa. 1999. Historia de los bosques relictos de "olivillo" (Aextoxicon punctatum) y Mirtáceas de la Isla Mocha, Chile, durante el Holoceno tardío. Revista Chilena de Historia Natural 72: 31-47.

Mendoza, G. \& V. Jerez. 2001. Coleópteros epígeos asociados a Peumus boldus Mol. en la Reserva Nacional Isla Mocha, Chile (Insecta, Coleoptera). Gayana 65: 129-136.

Parmesan, C. \& G. Yohe. 2003. A globally coherent fingerprint of climate change impacts across natural systems. Nature 421: 37-42.

Pefaur, J. \& J. YÁñEz. 1980. Ecología descriptiva de la Isla Mocha (Chile), en relación al poblamiento de vertebrados. Boletín Museo Nacional de Historia Natural 37: 107-112.

Quilhot, W., M. Cuellar, R. Díaz, F. Riquelme \& C. Rubio. 2010. Estudio preliminar de la flora liquénica de Isla Mocha, sur de Chile. Gayana Botánica 67: 206-212.

R Development Core Team. 2010. R: A language and environment for statistical computing. R. Foundation for Statistical Computing, Vienna, Austria. ISBN 3-900051 - 07-0, Url:
http://www.R-project.org.

Richardson, D.H.S. 1992. Pollution monitoring with lichens. Naturalists' Handbooks 19. Richmond Publishing Co., Ltd. Slough, England. 76 pp.

Richardson, D.H.S. \& R.P. CAMERON. 2004. Cyanolichens: their response to pollution and possible management strategies for their conservation in northeastern. Naturalist 11: 1-22.

Root, T.L., J.T. Price, K.R. Hall, S.H. Schneider, C. Rosenzweig \& J.A. Punds. 2003. Fingerprints of global warming on wild animals and plants. Nature: 421: 57-60.

Rose, C.I. 1976. Lichenological indicators of age and environmental continuity in woodlands. Lichenology: Progress \& Problems 8: 279-307.

Rose, F. 1992. Temperate Forest Management: its effect on bryophyte and flora and habitats. In: J.W. Bates \& A.M. Farmer (eds.), Bryophytes and Lichens in a Changing Environment, pp. 211- 233. Clarendon Press, Oxford.

Sillett, S. \& B. McCune. 1998. Survival and growth of cyanolichens transplants in douglas-fir forest canopies. The Bryologist 101: 20-31.

Stevens, D. 1994. Implementation of a National Monitoring Program. Journal of Environmental Management 42: 129.

Werth, S. 2001. Key factors for epiphytic macrolichen vegetation in deciduous forests of Troms county, northern Norway: human impact, substrate, climate or spatial variation? Unpublished candidate scientific thesis, University of Tromsø, Tromsø. 58 pp., i-xxxi.

Wirth, V. 1988. Phytosociological approaches to air pollution monitoring with lichens. In: T.H. Nash III (ed.), Lichens, Bryophytes and Air Quality. pp. 91-107.

Wirth, V. 1995a. Die Flechten: Baden -Württembergs. Tomo I. Editorial Verlag Eugen Ulmer. pp. 1-532.

Wirth, V. 1995b. Die Flechten: Baden -Württembergs. Tomo II. Editorial Verlag Eugen Ulmer. pp. 533-1006.

Recibido: 17.01.11

Aceptado: 14.07.11 